\title{
Benefits of spatiotemporal modeling for short-term wind power forecasting at both individual and aggregated levels
}

\author{
Lenzi, Amanda; Steinsland, Ingelin; Pinson, Pierre
}

Published in:

Environmetrics

Link to article, DOI:

10.1002/env.2493

Publication date:

2018

Document Version

Peer reviewed version

Link back to DTU Orbit

Citation (APA):

Lenzi, A., Steinsland, I., \& Pinson, P. (2018). Benefits of spatiotemporal modeling for short-term wind power forecasting at both individual and aggregated levels. Environmetrics, [e2493]. https://doi.org/10.1002/env.2493

\section{General rights}

Copyright and moral rights for the publications made accessible in the public portal are retained by the authors and/or other copyright owners and it is a condition of accessing publications that users recognise and abide by the legal requirements associated with these rights.

- Users may download and print one copy of any publication from the public portal for the purpose of private study or research.

- You may not further distribute the material or use it for any profit-making activity or commercial gain

- You may freely distribute the URL identifying the publication in the public portal 


\title{
Benefits of spatiotemporal modeling for short-term wind power forecasting at both individual and aggregated levels
}

\author{
Lenzi, Amanda; Pinson, Pierre; Steinsland, Ingelin \\ Published in: \\ Environmetrics
}

Publication date:

2019

Document Version

Early version, also known as pre-print

Link back to DTU Orbit

\section{Citation (APA):}

Lenzi, A., Pinson, P., \& Steinsland, I. (2019). Benefits of spatiotemporal modeling for short-term wind power forecasting at both individual and aggregated levels. Manuscript submitted for publication.

\section{General rights}

Copyright and moral rights for the publications made accessible in the public portal are retained by the authors and/or other copyright owners and it is a condition of accessing publications that users recognise and abide by the legal requirements associated with these rights.

- Users may download and print one copy of any publication from the public portal for the purpose of private study or research.

- You may not further distribute the material or use it for any profit-making activity or commercial gain

- You may freely distribute the URL identifying the publication in the public portal 


\section{Benefits of spatio-temporal modelling for short term wind power forecasting at both individual and aggregated levels}

\section{Amanda Lenzi ${ }^{a *}$ and Ingelin Steinsland ${ }^{b}$ and Pierre Pinson ${ }^{c}$}

Summary: The share of wind energy in total installed power capacity has grown rapidly in recent years. Producing accurate and reliable forecasts of wind power production, together with a quantification of the uncertainty, is essential to optimally integrate wind energy into power systems. We build spatio-temporal models for wind power generation and obtain full probabilistic forecasts from 15 minutes to 5 hours ahead. Detailed analysis of the forecast performances on the individual wind farms and aggregated wind power are provided. The predictions from our models are evaluated on a data set from wind farms in western Denmark using a sliding window approach, for which estimation is performed using only the last available measurements. The case study shows that it is important to have a spatio-temporal model instead of a temporal one to achieve calibrated aggregated forecasts. Furthermore, spatio-temporal models have the advantage of being able to produce spatially out-of-sample forecasts. We use a Bayesian hierarchical framework to obtain fast and accurate forecasts of wind power generation at wind farms where recent data are available, but also at a larger portfolio including wind farms without recent observations of power production.

The results and the methodologies are relevant for wind power forecasts across the globe as well as for spatial-temporal modelling in general.

Keywords: wind power; aggregated forecast; probabilistic forecast; integrated nested Laplace approximation.

\footnotetext{
${ }^{\mathrm{a}}$ Applied Mathematics and Computer Science Department, Technical University of Denmark, 2800 Kgs. Lyngby, Denmark

${ }^{\mathbf{b}}$ Department of mathematical sciences, Norwegian University of Science and Technology, N-7491 Trondheim, Norway

${ }^{\mathbf{c}}$ Electrical Engineering Department, Technical University of Denmark, 2800 Kgs. Lyngby, Denmark

* Correspondence to: Applied Mathematics and Computer Science Department, Technical University of Denmark, 2800 Kgs. Lyngby,

Denmark.E-mail: lenzi.amanda88@gmail.com
}

This paper has been submitted for consideration for publication in Environmetrics 


\section{INTRODUCTION}

Wind power is a clean, renewable and widely available source of energy and electricity generated from wind power is increasing world wide. A challenge for utilizing wind power is that the generated amount of energy varies much and relatively fast over time due to variations in wind. An important tool for efficiently integrating wind power in a system with energy sources that can be controlled, e.g. thermal energy and hydro power, is high quality probabilistic forecasts for short term wind power production [Ackermann, 2005]. Recently, there has been an increasing amount of research in wind speed and wind power forecasts. Most of the developments are for point forecasts (e.g. Louka et al. [2008], Catalão et al. [2011]), i.e. the forecast consists of one value for each wind farm or location. To make better decisions one also needs to quantify the uncertainty of the forecast, and provide a probability density function (pdf) instead of a point forecast. This is called a probabilistic forecast. For a probabilistic forecast to be useful it needs to be calibrated [Gneiting et al., 2007]. Calibrated refers to a forecast that is reliable: in the long term, $90 \%$ of the observed wind production should be within a $90 \%$ forecast interval, $80 \%$ of the observations within a $80 \%$ forecast interval and so forth.

In recent years, more emphasis has been placed on probabilistic forecasts in order to quantify the inherent uncertainties in wind, see Pinson and Kariniotakis [2010] and Bremnes [2004]. The advantage of using probabilistic forecasts, compared to point forecasts, has been demonstrated in several applications. Indeed, probabilistic forecasts are the optimal input to a large class of decision-making problems, where the optimal bid is not the expected power but a quantile [Gneiting, 2011]. For instance, probabilistic methods are already widely used among system operators to address decision-making problems related to reserve quantification [Matos and Bessa, 2011], [Garver, 1966] and unit commitment considering wind power uncertainty [Papavasiliou and Oren, 2013], [Wang et al., 2011]. Additionally, quantifying the uncertainties in the forecasts helps define advanced strategies for market 
participation, which in turn reduce imbalance charges to producers making wind power more competitive in the energy market, and maximize the profit of energy providers with the development of bidding strategies [Girard et al., 2013]. From the point of view of a system operator, the aggregated wind power generation over pre-defined areas is of particular importance. Some recent contributions to the modelling and forecasting of aggregated wind power energy are Lau and McSharry [2010] and Focken et al. [2002], which do, however, not account for spatio-temporal dependencies.

To illustrate the challenge of forecasting individual and aggregated wind power simultaneously, we consider a toy example of two wind farms at one lead time and denote their forecasts $X_{1}$ and $X_{2}$ (these are random variables). The aggregated forecast for the system is $Y=X_{1}+X_{2}$. We know from basic probability, see e.g Ross [2015], that the expected value for the system is $\mathrm{E}(Y)=\mathrm{E}\left(X_{1}\right)+\mathrm{E}\left(X_{2}\right)$ and the variance is $\operatorname{Var}(Y)=\operatorname{Var}\left(X_{1}\right)+\operatorname{Var}\left(X_{2}\right)+2 \operatorname{Cov}\left(X_{1}, X_{2}\right)$. Hence, to obtain a forecast for the system $Y$ we also need to model the dependency between the wind farms. This calls for a spatiotemporal model for wind power production. If the productions at the two farms in our toy example are dependent and have a positive covariance, but are assumed independent in the forecast, the variance of $Y$ gets too small and the forecast for $Y$ is not calibrated. Verification of multivariate probabilistic forecasts is an active field of research, for which new scores and diagnostic tools are being proposed and discussed, see, e.g., Pinson and Tastu [2013], Scheuerer and Hamill [2015], Thorarinsdottir et al. [2016] among others. A pragmatic approach is to evaluate relevant univariate probabilistic forecasts derived from the multivariate probabilistic forecast.

Understanding a spatio-temporal process throughout a country, such as wind power production in Denmark, is a difficult problem due to the complex temporal and spatial structures. This explains why there is very little research on large wind power data sets and most of the prediction systems are optimized for each and every location individually 
without taking the spatio-temporal dependency into consideration. Generally speaking, several approaches exist for modelling spatio-temporally correlated data [Cressie, 2015] [Blangiardo and Cameletti, 2015]. A method based on temporal basis functions to account for the temporal correlation and spatially varying coefficients to account for spatial variability was used in Lindström et al. [2014] to model ambient air pollution and is available for implementation in the R package "SpatioTemporal" [Lindström et al., 2013]. Another widely used approach focuses on modelling the spatio-temporal covariance function, which summarizes many aspects of a process. While there has been considerable development on classes of spatio-temporal covariance functions recently (see e.g. Gneiting and Guttorp [2010]), in practice, it is common to propose simplifying assumptions, such as separability, in order to estimate the covariance function in a feasible way. In the following, we use a relatively simple covariance model that can be re-trained periodically in the same way with low computational cost. In this way it is possible to have a spatio-temporal predictor for hundreds of wind farms with an automated model fitting scheme, which is essential given the abundance of wind farms in power systems today. To our knowledge, this is the first attempt to date to address Bayesian hierarchical models for obtaining spatio-temporal forecasts of wind power generation.

Several characteristics in a typical wind power series make it a challenging problem to generate accurate forecasts. First of all, wind power is bounded below by zero, when no turbines are operating, and above by the nominal capacity, when all turbines are generating their rated power output. In addition, wind power series are clearly non-Gaussian. Instead of using a classical Gaussian distribution, truncated Gaussian, censored Gaussian and generalized logit-normal distributions have been proposed to model the conditional density of wind power [Gneiting et al., 2006], [Pinson, 2012]. Our approach is based on the logistic function, which has shown to be a suitable transformation to normalize wind power data [Dowell and Pinson, 2016]. 
We propose statistical models that yield calibrated probabilistic forecasts of wind power generation at multiple sites and lead times simultaneously. We define three different models that share the same data process, or likelihood, but differ in the process model. We start with a model consisting of a location specific intercept and an autoregressive component that captures the local variability without considering the dependency between the farms. This model is well suited for individual forecasts, but it is not calibrated for aggregated forecasts. To obtain reliable aggregated forecasts, we introduce two different models that capture the spatio-temporal features present in the data. The first has a common intercept and a spatio-temporal process, in which spatial and temporal dependency is modelled by a latent Gaussian field. The second is a combination of the previous two models, with a common intercept, an autoregressive process and a spatio-temporal term that varies in time with first order autoregressive dynamics. To meet the computational requirements a stochastic partial differential equations (SPDE) approach to spatial and temporal-spatial modelling is taken [Lindgren et al., 2011], [Blangiardo and Cameletti, 2015], for which fast Bayesian inference can be performed using integrated nested Laplace approximations (INLA).

Moreover, we study the performance of the proposed models in forecasting wind power from individual and aggregated farms under two different scenarios. In a evaluation scheme, we consider out-of-sample forecasts in terms of time, that is, they are obtained for wind farms inside the training set. However, there are situations where not enough data is available for all the wind farms, and even when it is available, the computational load to calculate forecasts for all of them can be very high. In those cases, spatial interpolation methods are used to obtain predictions for the whole system based only on part of the power production observations. For an energy producer, spatial prediction is of relevance for a number of operational problems, where wind power generation is only observed at a limited number of wind farms, while decision-making problems may require an overview of power generation at all sites over a region. Based on this, in a second evaluation scheme, we consider spatially 
out-of-sample forecasts generated by the proposed spatio-temporal models. For both schemes we develop and evaluate the forecasts for wind power production in western Denmark based on a data set for 349 wind farms with energy production observations every 15 minutes from 2006 to 2012.

In Section 2, we provide a short description of the wind power data that we use in our study and the data treatment. The hierarchical models used to generate probabilistic forecasts of wind power generation, as well as the framework for producing probabilistic forecasts with such models, are outlined in Section 3. In Section 4, we give details of the probabilistic forecasting scheme and outline the scores and the scenarios used for forecast evaluation. In Section 5, we show the results of a case study where we obtain spatio-temporal forecasts and spatially out-of-sample forecasts on the individual and aggregated level. Conclusions of the work are drawn in Section 6.

\section{DANISH WIND POWER PRODUCTION DATA}

This project is based on a system of 349 wind farms in western Denmark. Observations of wind power production between January 2006 and March 2012 were provided by the Transmission System Operator in Denmark and each measurement consists of temporal average over a 15-min time period.

The measurements at each site have been normalised by the nominal power of the corresponding wind farm, so that they are within the range $[0,1]$. Moreover, to avoid including long chains of zeros that come from temporary shutdown of the turbines for maintenance or missing data that are reflected as unreasonably long periods of zero wind power production, we choose to analyze only wind farms containing at most $10 \%$ of zero observations. The evaluation of the predictive performance of individual wind farms and aggregated wind power is done as $\%$ of nominal power, which is a common practice in the wind power field 
(e.g., Pinson [2012], Tastu et al. [2011], Dowell and Pinson [2016]).

Figure 1 (a) shows the spatial correlation of wind power production between one wind farm located in the southern part of Denmark and the remaining wind farms of the portfolio. The higher correlations come from farms that are closer, while the correlations of wind farms far from it are almost zero. Next, we check the dependency of the temporal correlation at fixed locations. Figure 1 (b) shows the mean autocorrelation function of wind power production among wind farms located in western Denmark. The autocorrelation function of the normalized wind power production at a single farm has a slow decay and on average, it drops down to zero after about 40 hours.

Since our aim is to generate short term forecasts (from 15 min up to 5 hours ahead), we do not focus on modeling any daily or long term seasonality, which often appears in wind data due to different wind patterns throughout the day or the year. One should notice that even though the proposed models can be used to generate predictions at any given time during the year, for the particular application, we use only the last two days of data to fit the models (see Section 4.1) where no seasonality can be detected.

[Figure 1 about here.]

Figure 2 (a) shows examples of normalized wind power production from the start of June 1 to the end of June 2 at four different wind farms, while Figure 2 (b) shows their locations on the map of Denmark. It is clear that wind farms that are close to each other have similar pattern of power production. The two northeastern farms (blue and red circles) have power production close to zero at the first day of June and a peak at around noon of June 2, while the wind farms located at the southwest part of the island (green and pink circles) have larger power generation at all times, with the peak at the end of June 2.

[Figure 2 about here.] 
Wind power generated by a farm over a period of time is non-Gaussian and bounded between zero and one after the normalization. In fact, wind power distribution has a sharper peak than the Gaussian distribution and is also significantly right-skewed. In all the approaches to be described next, we apply the logit-normal transformation to the normalized wind power data following the procedure in Pinson [2012].

Let $X(\mathbf{s}, t)$ denote the normalized wind power production at location $\mathbf{s} \in \mathcal{D}_{s}$ and time $t \in \mathcal{D}_{t}$, with respective observations or measurements indicated by $x(\mathbf{s}, t)$. The logit-normal transformation is given by

$y(\mathbf{s}, t)=\gamma(x(\mathbf{s}, t))=\ln \left(\frac{x(\mathbf{s}, t)}{1-x(\mathbf{s}, t)}\right), \quad x(\mathbf{s}, t) \in(0,1)$

with inverse

$x(\mathbf{s}, t)=\gamma^{-1}(y(\mathbf{s}, t))=\left(1+e^{-y(\mathbf{s}, t)}\right)^{-1}, \quad y(\mathbf{s}, t) \in \mathbb{R}$.

Similarly to the approach of Lesaffre et al. [2007] for modelling outcomes in [0, 1], we define a threshold $\epsilon$ to represent the logit-normal transformation at the bounds. Wind power values less than $\epsilon$, or greater than $1-\epsilon$, are considered to be 0 or 1 , respectively.

Moreover, to evaluate the performance of aggregated wind power forecasts, we obtain the normalized aggregated wind power at lead time $h$ by

$x_{A}(t+h)=\frac{\sum_{j=1}^{N} c_{j} x\left(\mathbf{s}_{j}, t+h\right)}{\sum_{j=1}^{N} c_{j}}$,

where $c_{j}$ is the capacity of wind farm at location $\mathbf{s}_{j}$ and $N$ is the total number of wind farms in the portfolio.

\section{MODELS AND FITTING SCHEME}


Since wind power is affected by a number of physical factors that depend, for instance, on changes of season and climate, it is to be expected that the parameters of the model change slowly over time. Therefore, it is appropriate to either allow the parameters of the models to track this variation, or to model it directly. In terms of spatial dependence, since prevailing wind patterns propagate along certain directions, space is usually no longer homogeneous over all directions. In the case of Denmark, although there is anisotropy in the wind power data, we noticed that the main direction of the wind changes depending on the days. For instance, while there is a dependence of the correlation on the direction west to east on the first two days of January, this dependence is stronger from north to south when looking at the next two days. To allow the parameters to track these variations by changing according to the data set that has been modelled, we use a sliding window approach with a relatively simple covariance model that can be re-trained in the same way periodically with low computational cost.

In this section, we introduce three different statistical models for wind power production. We start with a simpler autoregressive model, where each wind farm is considered as an independent replicate of the same process. Next, we describe two versions of a spatiotemporal model, in which spatial correlation is captured by a latent Gaussian field with a Matérn covariance function. The simplest version has only a spatio-temporal component, while the other has both, an autoregressive process and a spatio-temporal model. The section ends with the estimation procedure and how we obtain probabilistic forecasts.

\subsection{Likelihood}

We denote by $Y(\mathbf{s}, t)$ the normalized logit-normal transformed wind power generation at location $\mathbf{s}$ and time $t$, which is calculated using (1). We assume the following distribution 
for $Y(\mathbf{s}, t)$ at the first level of the hierarchical models considered in this section

$Y(\mathbf{s}, t) \sim \operatorname{Normal}\left(\mu(\mathbf{s}, t), \sigma_{e}^{2}\right)$

with $\sigma_{e}^{2}$ being the variance of a Gaussian white noise both serially and spatially uncorrelated. We assume that $\mu(\mathbf{s}, t)$ is latent, since it is observed only with a noise term, and can be defined by other process levels giving rise to different hierarchical models that are described in the following sections.

\subsection{Latent Gaussian structure}

\subsubsection{Temporal model (Model T)}

We start with a time series model where each wind farm is considered as an independent replicate of the same random process. The independence assumption is of course a simplification, since the wind power production in one location is probably dependent on the production in other locations. We assume that $\mu(\mathbf{s}, t)$, in $(4)$, is constant in time and can be modelled as

$\mu(\mathbf{s}, t)=b(\mathbf{s})+w_{\mathbf{s}}(t)$

where $b(\mathbf{s})$ is an intercept specific for each location and $w_{\mathbf{s}}(t)$ is an autoregressive process that can be written as

$w_{\mathbf{s}}(t)=\rho_{1} w_{\mathbf{s}}(t-1)+\nu_{\mathbf{s}}(t)$

with $t=2, \ldots, T$ and $\left|\rho_{1}\right|<1$. The term $\nu_{\mathbf{s}}$ is uncorrelated with $w_{\mathbf{s}}(t)$ and independent identically distributed as $\nu_{\mathbf{s}} \sim N\left(0, \sigma_{\nu}^{2}\right)$. 


\subsubsection{Spatio-temporal model (Model S-T)}

This model is a spatio-temporal process with temporal dynamics as in Cameletti et al. [2013]. This type of model has been used for modelling air quality because of its flexibility in including time and space dependency, as well as the effect of covariates (see e.g. Fassò and Finazzi [2011] and Cocchi et al. [2007]). The mean function $\mu(\mathbf{s}, t)$ in (4) is given by

$\mu(\mathbf{s}, t)=b_{0}+z(\mathbf{s}, t)$

where $b_{0}$ is an intercept that is common to all wind farms and constant in time and space. The term $z(\mathbf{s}, t)$ refers to a spatio-temporal process that varies in time with first order autoregressive dynamics

$z(\mathbf{s}, t)=\rho_{2} z(\mathbf{s}, t-1)+w(\mathbf{s}, t)$

with $t=2, \ldots, T$ and $\left|\rho_{2}\right|<1$. Moreover, $w(s, t)$ is a zero-mean Gaussian field, assumed to be temporally independent with covariance function

$\operatorname{Cov}\left(w(\mathbf{s}, t), w\left(\mathbf{s}^{\prime}, t^{\prime}\right)\right)= \begin{cases}\sigma_{w}^{2} C(h), & \text { if } t=t^{\prime} \\ 0, & t \neq t^{\prime}\end{cases}$

for $\mathbf{s} \neq \mathbf{s}^{\prime}$. The correlation function $C$ depends on the locations $\mathbf{s}$ and $\mathbf{s}^{\prime}$ through the distance $h=\left\|\mathbf{s}-\mathbf{s}^{\prime}\right\|$. This means that the process is assumed to be second-order stationary and isotropic (see Cressie [1992]). The marginal variance is $\operatorname{Var}(\mathbf{s}, t)=\sigma_{w}^{2}$ and $C(h)$ is the correlation function defined by the Matérn, given by

$C(h)=\frac{1}{\Gamma(\nu) 2^{\nu-1}}(\kappa h)^{\nu} K_{\nu}(\kappa h)$, 
where $K_{\nu}$ is the modified Bessel function of second kind, order $\nu$. The parameter $\kappa$ can be used to select the range, while $\nu$ is a smoothness parameter that can be used to determine the mean-square differentiability of the underlying process. More precisely, the range is defined to be $r=\sqrt{8 \nu} / \kappa$ while the marginal variance is $\sigma_{w}^{2}=1 /\left(4 \pi \tau^{2} \kappa^{2}\right)$. Although the parameter $\nu$ is fixed to 1 for computational reasons, it remains flexible enough to handle a broad class of spatial variation Rue et al. [2009]. Applications with fixed parameter $\nu$ include Ingebrigtsen et al. [2014], Cameletti et al. [2013] and Munoz et al. [2013].

\subsubsection{Temporal + Spatio-temporal model (Model ST+T)}

This is a model defined by an autoregressive process at each location to capture the individual variability and a spatio-temporal process with temporal dynamics to take into account the spatial dependence among wind farms. Specifically, $\mu(\mathbf{s}, t)$ from (4) is defined as

$\mu(\mathbf{s}, t)=b_{0}+w_{\mathbf{s}}(t)+z(\mathbf{s}, t)$,

where $b_{0}$ is a fixed unknown intercept that is shared by all wind farms. The process $w_{\mathbf{s}}(t)$ is assumed to have autoregressive dynamics as defined in (6). Finally, $z(\mathbf{s}, t)$ is a spatio-temporal component that has the structure of (8) and its spatio-temporal covariance function is the same as in (9).

\subsubsection{Prior specification}

For the intercepts a flat prior (uniform) is used. The correlations $\rho$ 's are specified through $\beta=\log \left(\frac{1+\rho}{1-\rho}\right)$, and $\beta$ has a Gaussian prior distribution with zero mean and precision 0.15. A log-Gamma prior with parameters 1 and 0.00005 is assumed for $\log \left(\sigma_{e}^{2}\right)$ and $\log \left(\sigma_{\nu}^{2}\right)$. The priors for the parameters in the Matérn field, $\kappa$ and $\tau$, are specified with the parameterization $\log (\kappa)$ and $\log (\tau)$ as Gaussian distributed. The prior mean for $\log (\kappa)$ is chosen heuristically 
so that the range of the field is about $20 \%$ of the diameter of the region, while the prior mean for $\log (\tau)$ is chosen so that the corresponding variance of the field is 1 . Finally, the prior precisions of $\log (\kappa)$ and $\log (\tau)$ are set to 0.1 .

\subsection{Inference and prediction}

The key feature of the models described above is that they can be handled within the theoretical and computational framework developed by Rue et al. [2009] and Lindgren et al. [2011]. Rue et al. [2009] introduced integrated nested Laplace approximations (INLA) that allows us to directly compute accurate and fast approximations of the posterior marginals for latent Gaussian Markov random field (GMRF) models. A key feature is the utilization of the sparseness of the precision matrix (inverse covariance matrix). For spatial and spatialtemporal modelling, Gaussian random fields (GRF) models are popular choices, see Cressie [2015]. Lindgren et al. [2011] introduced a link between latent GRF models and GMRF models: modeling can be done using latent GRF models (with dense covariance and precision matrices), while computations are carried out with GMRFs with a sparse precision matrix. The original idea comes from the work of Whittle [1954] and Whittle [1963], where it is shown that the only stationary solution to the SPDE

$\left(\kappa^{2}-\Delta\right)^{\alpha / 2} \eta(\mathbf{s})=\mathcal{W}(\mathbf{s}), \quad \mathbf{s} \in \mathbb{R}^{d}, \alpha=\nu+D / 2, \kappa>0, \nu>0$

is a GRF with Matérn covariance function. The innovation process $\mathcal{W}$ on the right hand side of (11) is Gaussian white noise and $\Delta$ is the Laplacian.

An approximation to the solution of the SPDE in (11) can be obtained using the finite element method (FEM), a numerical technique for solving partial differential equations [Lindgren et al., 2011]. This is done by representing the infinite dimensional GRF by a 
linear combination of finite basis function

$\eta(\mathbf{s})=\sum_{k} \psi_{k}(\mathbf{s}) w_{k}$

where the $w_{k}$ 's are random weights chosen so that the representation in (12) approximates the distribution of the solution to the SPDE in (11). The random weights form a GMRF and the $\psi_{k}$ 's are basis functions defined on a triangulation of the domain, i.e. a subdivision into non-intersecting triangles. Figure 3 shows the triangulation of western Denmark data set described in Section 2.

[Figure 3 about here.]

Next, the posterior estimates of the model parameters, which we will denote by $\pi(\boldsymbol{\theta} \mid \mathbf{y})$, are computed using INLA [Rue et al., 2009]. This method approximates the integral involved in the calculation of the marginal posterior distributions of the parameters by Laplace approximation, making use of the Markov structure of the latent variables in the computation. We use the R-INLA package to perform inference and prediction. For more information on the package see http://www.r-inla.org.

In addition to the marginal distribution of the model parameters, one can also get marginal posteriors for linear combinations of variables in the GMRF. For non-linear functions one can base approximations on Monte Carlo sampling. This is achieved by first sampling from the posterior of the parameters $\pi(\boldsymbol{\theta} \mid \mathbf{y})$, followed by sampling from the Gaussian approximation of the latent field $\pi(\boldsymbol{\eta} \mid \mathbf{y}, \boldsymbol{\theta})$. For Gaussian likelihoods, the Laplace approximation is exact and the samples are directly computed from the posterior distribution. These samples can then be used to obtain the sum of the linear predictors through the build-in function inla.posterior. sample. 


\section{FORECAST EVALUATION}

\subsection{Probabilistic forecasting scheme}

We evaluate the predictive performance of the models described in Section 3 using a time moving window approach with data from western Denmark in 2009, so that we consider each wind farm as a training set with length $L=2 \times 96=192$ observations, i.e., two days. In total, the model is fit to $364 / 2=182$ different data sets. We obtain forecasts for lead times $h=1, \ldots, 20$, that is, from 15 minutes up to 5 hours following the training data. Notice that we have compared different lengths of data window $L$ with respect to the root mean squared error (RMSE) and continuous ranked probability score (CRPS). Model T is very sensitive to the window length, such that less than two days of observations in the training set resulted in poor estimation at all lead times. On the other hand, Model S-T and Model ST+T showed to be robust for different values of $L$, with small changes in the forecast performance for different training sets.

Moreover, because of the high-time resolution of the Danish wind power time series (15minutes) and the dependency structure in space and time of Model S-T and Model ST+T, the fitting can be very computationally expensive. One way to deal with high-time resolution data is to define the model on a set of knots instead of all time points. Knot-based linear combinations are widely used to tackle computational problems in large data sets (e. g. Paciorek [2007] and Wikle and Cressie [1999]). To fit the spatio-temporal component $z(\mathbf{s}, t)$ in (8), we define a set of equally spaced knots at every 12 data points (3 hours), such that the points in time are reduced to only 17 knots, instead of the original 192 observations. Note that the component $w_{\mathbf{s}}(t)$ in models Model $\mathrm{T}$ and Model $\mathrm{ST}+\mathrm{T}$ is fitted to the complete training data, since it does not involve spatio-temporal interactions.

We evaluate probabilistic forecasts of wind power production from individual wind farms and aggregated. 
Let $\hat{X}\left(\mathbf{s}_{j}, t+h\right)$ denote the random variable of the wind power forecast at wind farm $\mathbf{s}_{j}$ and lead time $h$. The aggregated forecast of wind power generation is taken as

$$
\hat{X}_{A(t+h)}=\frac{\sum_{j=1}^{N} c_{j} \hat{X}\left(\mathbf{s}_{j}, t+h\right)}{\sum_{j=1}^{N} c_{j}}
$$

where $c_{j}$ is the capacity of wind farm $\mathbf{s}_{j}$ and $N$ is the number of wind farms. To find

the pdf of the aggregated forecasts, $\hat{f}_{X_{A(t+h)}}$, the joint distribution for all wind farms $\left\{\hat{X}\left(\mathbf{s}_{1}, t+h\right), \hat{X}\left(\mathbf{s}_{2}, t+h\right), \ldots \hat{X}\left(\mathbf{s}_{N}, t+h\right)\right\}$ needs to be assessed. Finally, point forecast of aggregated wind power production is obtained as the mean (or median) of $\hat{f}_{X_{A(t+h)}}$.

The aggregated wind power forecast in (13) is a sum over non-linear transformations of the predicted transformed wind power production. To access the predicted aggregated forecast, we sample from the joint posterior of the parameters and compute the expected transformed wind power production. For each wind farm, we add an independent identically distributed Gaussian error with mean zero and variance equal to the sample from the posterior distribution of $\sigma_{\epsilon}^{2}$. For each sample and each wind farm, the corresponding accumulated production is then calculated using (13).

\subsection{Point and probabilistic forecast scores}

We assess the quality of predictive performance of the models proposed in Section 3 using both point and probabilistic forecast scores. We obtain point forecast at a specific location as the mean of the forecast density. For each lead time, point forecast of individual power is assessed using the root mean squared error (RMSE), where the mean is taken over all wind farms and data sets,

$\operatorname{RMSE}(t+h)=\sqrt{\frac{1}{D N} \sum_{i=1}^{D} \sum_{j=1}^{N}\left(x\left(\mathbf{s}_{i j}, t+h\right)-\hat{x}\left(\mathbf{s}_{i j}, t+h\right)\right)^{2}}$ 
where $x\left(\mathbf{s}_{i j}, t+h\right)$ is the normalized wind power production at wind farm $\mathbf{s}_{j}$ of data set $i$ and lead time $h$. The term $\hat{x}\left(\mathbf{s}_{i j}, t+h\right)=\gamma^{-1}\left(\hat{y}\left(\mathbf{s}_{i j}, t+h\right)\right)$ is the predicted value of $x\left(\mathbf{s}_{i j}, t+h\right)$.

To evaluate the performance of forecast densities, we use the continuous ranked probability score (CRPS). Gneiting and Raftery [2007] showed that CRPS is a strictly proper scoring rule for the evaluation of probabilistic forecasts of a univariate quantity that assesses calibration and sharpness simultaneously Gneiting and Raftery [2007]. A lower score indicates a better density forecast. It is defined as

$\operatorname{CRPS}(F, x)=\int_{-\infty}^{\infty}\left(F(y)-\delta_{\{y \geq x\}}\right)^{2} d y$

where $\delta_{\{A\}}$ is the indicator function that is equal to one when property $\mathrm{A}$ is satisfied and zero otherwise, $F$ is the cumulative distribution function of the density forecast and $y$ is the observation. With the available samples, we can approximate the mean CRPS at each lead time by

$$
\begin{aligned}
\operatorname{CRPS}_{F, x}(t+h)= & \frac{1}{D N} \sum_{i=1}^{D} \sum_{j=1}^{N}\left(\frac{1}{n} \sum_{k=1}^{n}\left|\hat{x}^{(k)}\left(\mathbf{s}_{i j}, t+h\right)-x\left(\mathbf{s}_{i j}, t+h\right)\right|\right. \\
& \left.-\frac{1}{2 n^{2}} \sum_{k, l=1}^{n}\left|\hat{x}^{(k)}\left(\mathbf{s}_{i j}, t+h\right)-\hat{x}^{(l)}\left(\mathbf{s}_{i j}, t+h\right)\right|\right),
\end{aligned}
$$

where $\mathrm{n}$ is the number of samples. Again, the mean CRPS is taken over all the wind farms and data sets in the training set.

Reliability, also referred to as calibration, of probabilistic forecasts is assessed with reliability diagrams. In a calibrated forecast, the observed levels should match the nominal levels for specific quantile forecasts, which results in points aligning with the diagonal in the reliability diagram. To construct reliability diagrams, we start by introducing an indicator variable $\mathcal{I}^{(\alpha)}\left(\mathbf{s}_{i j}, h\right)$, which is defined for a quantile forecast $\hat{q}^{(\alpha)}\left(\mathbf{s}_{i j}, t+h\right)$ issued at lead time 
$h$ and wind farm $\mathbf{s}_{i}$ of the training data $j$, with observed value $x\left(\mathbf{s}_{i j}, t+h\right)$ as follows

$\mathcal{I}^{(\alpha)}\left(\mathbf{s}_{i j}, h\right)= \begin{cases}1 & \text { if } \quad x\left(\mathbf{s}_{i j}, t+h\right) \leq \hat{q}^{(\alpha)}\left(\mathbf{s}_{i j}, t+h\right) \\ 0, & \text { otherwise }\end{cases}$

The indicator variable $\mathcal{I}^{(\alpha)}\left(\mathbf{s}_{i j}, h\right)$ shows whether the actual outcome lies below the $\alpha$ quantile forecast (hits) or not (miss). Next, $n_{h, 1}^{(\alpha)}$ denotes the sum of hits and $n_{h, 0}^{(\alpha)}$ the sum of misses over all the realizations

$n_{h, 1}^{(\alpha)}=\sum_{i=1}^{D} \sum_{j=1}^{N} \mathcal{I}^{(\alpha)}\left(\mathbf{s}_{i j}, h\right) \quad$ and $\quad n_{h, 0}^{(\alpha)}=D N-n_{h, 1}^{(\alpha)}$.

An estimation $\hat{a}_{h}^{(\alpha)}$ of the actual coverage $a_{h}^{(\alpha)}$ is then obtained by calculating the mean of $\mathcal{I}^{(\alpha)}\left(\mathbf{s}_{i j}, h\right)$ over the $N$ wind farms in the $D$ validation sets

$\hat{a}_{h}^{(\alpha)}=\frac{1}{D N} \sum_{i=1}^{D} \sum_{j=1}^{N} \mathcal{I}^{(\alpha)}\left(\mathbf{s}_{i j}, h\right)=\frac{n_{h, 1}^{(\alpha)}}{n_{h, 1}^{(\alpha)}+n_{h, 0}^{(\alpha)}}$.

Here, we use nominal levels from $5 \%$ to $95 \%$ in steps of $5 \%$. Since the number of observations used to calculate the reliability diagrams is of limited size and the observed proportions are equal to the nominal ones only asymptotically Toth et al. [2003] Bröcker and Smith [2007], we follow the idea of Bröcker and Smith [2007] of generating consistency bars for reliability diagrams.

\subsection{Evaluation scheme}

We evaluate probabilistic forecasts of Danish wind power production from two different scenarios. First, we consider time forward forecast performances at the locations of the training set. The spatio-temporal models, i.e, Model S-T and Model ST+T, have the advantage of being able to provide forecasts where recent observations are not available. 
Based on this, in a second evaluation scheme, we study the performances of spatially out-ofsample forecasts, which are based on $k$-fold cross-validation with $k=5$. Notice that overall, 5 to 10 -fold cross-validation is recommended as a good compromise between bias and variance (Breiman and Spector [1992]; Kohavi et al. [1995]). The forecast performance measures from the second scenario are obtained by combining the estimates from the 182 data sets in the training set.

\section{RESULTS}

In this section we show the results from a case study, where we use the models described in Section 3 to forecast individual and aggregated wind power in Denmark. As described in Section 4.3, we evaluate and discuss the performances of our models when we consider time forward forecasts at the locations of the training set. We call these spatio-temporal forecasts, and we also show the case of spatially out-of-sample forecasts, i.e, for wind farms that are not in the training set. Details of the probabilistic forecasting scheme can be found in Section 4.1, while the methodology used to rank point and probabilistic forecasts is in Section 4.2 .

\subsection{Spatio-temporal forecast performance}

Figure 4 summarizes the spatio-temporal forecast performances of the three models introduced in Section 3 in terms of RMSE and CRPS. As we can see from Figure 4 (a), Model T and Model ST+T outperformed Model S-T with respect to RMSE and CRPS when forecasting individual wind farms at lead times 1-6 (i.e, from 15 minutes up to 2 hours ahead). For higher lead times, the three models have similar performance. In terms of aggregated wind power production, Model $\mathrm{T}$ performed similar to Model $\mathrm{ST}+\mathrm{T}$ in terms of 
point forecast (RMSE), but it has poor performance according to CRPS values, as shown in Figure $4(\mathrm{~b})$.

Reliability diagrams for each model at lead times $h=1,7,13$ and 19 are presented in Figure 5. These diagrams compare the theoretical and the observed proportions of a set of quantiles from forecasts made at all wind farms and data sets in the training set. The forecasts at individual wind farms produced by the three models presented in Section 3 perform similarly well in terms of reliability, with points close to the diagonal for most quantiles, see Figure 5 (a). Since the number of observations used to calculate reliability diagrams is relatively small (182 data sets in the training set), consistency bars for the evaluation of forecasts from aggregated farms are also plotted, as shown in Figure 5 (b). The aggregated forecasts provided by Model ST+T are the best calibrated among the three models for most of the quantiles at all lead times, followed by Model S-T. Even though the performance of Model $\mathrm{T}$ is comparable with the performance of the other models in terms of aggregated forecast density mean (RMSE), we can see that this model does not produce reliable probabilistic forecasts for the aggregated data. This fact is more obvious for the lower quantiles; more than $50 \%$ of the observed aggregated forecasts are below the nominal $5 \%$ quantile at lead times $h=7,13$ and 19 .

[Figure 4 about here.]

[Figure 5 about here.]

We further explore aggregated probabilistic forecasts from models in Section 3 with plots containing the 5\%,50\% and 95\% quantiles of the aggregated forecast densities together with the actual observed aggregated power produced at four different data sets in the training set, as shown in Figure 6. We noticed that Model $\mathrm{T}$ results in forecast densities that are consistently too narrow. On the other hand, Model ST $+\mathrm{T}$ provides the widest aggregated forecast densities among the three models in most of the data sets, which produces calibrated forecasts at all lead times, as confirmed in Figure 5 (b). 
[Figure 6 about here.]

\subsection{Spatially out-of-sample forecast performance}

Figure 7 shows the out-of-sample forecast performances in terms of RMSE and mean CRPS for individual wind farms (a) and aggregated wind power (b). They are computed as the mean of the RMSE and CRPS from the 5-fold cross validations as described in Section 4.3. It can be seen that Model ST+T outperforms Model S-T at all lead times when predicting wind power at individual wind farms under RMSE and CPRS. When looking at aggregated out-of-sample forecasts, while for shorter lead times than 2 hours, Model S-T is better than Model ST+T in terms of RMSE, for longer horizons, Model ST+T out-performs Model S-T under the same score. In terms of CRPS, Model ST+T produces better aggregated forecasts at lead times 1-20 (i.e., from 15 minutes to 5 hours ahead).

Reliability diagrams at lead times $h=1,7,13$ and 19 are presented in Figure 8. We observe from Figure 8 (a) that Model S-T and Model ST+T provide relatively well calibrated forecast densities for individual farms. In terms of aggregated forecasts, we can see from Figure 8 (b) that Model ST $+\mathrm{T}$ is calibrated, since the line is always within the consistency bars. On the other hand, aggregated forecast densities obtained with Model S-T are poorly calibrated for quantiles lower than 0.75 . Indeed, $20 \%$ of the observations are below the $5 \%$ forecast quantile at lead times 1, 7, 13 and 19 .

[Figure 7 about here.]

[Figure 8 about here.]

\section{CONCLUSIONS}

In this article we have presented hierarchical spatio-temporal models for obtaining probabilistic forecasts of wind power generation at multiple locations and lead times. We 
started with a time series model consisting of an autoregressive process with a location specific intercept. The results for individual probabilistic forecasts were satisfactory in terms of skill scores and reliability, however, the aggregated probabilistic forecasts were not calibrated. After finding the unsatisfactory results for the reliability of aggregated forecasts, we introduced two different spatio-temporal models. The first has a common intercept for all farms and a spatio-temporal model that varies in time with first order autoregressive dynamics and has spatially correlated innovations given by a zero mean Gaussian process with Matérn covariance. The second model has a common intercept, an autoregressive process to capture the local variability and the spatio-temporal term. To deal with the non-Gaussianity of wind power series, a parametric framework for distributional forecasts based on the logit-normal transformation was used.

In a case study, the proposed models have been used to produce probabilistic forecasts of wind power at wind farms in western Denmark from 15 minutes up to 5 hours ahead for a test period of one year. Using an SPDE approach that is implemented in the R-INLA library, we obtained fast and accurate forecasts of wind power generation at wind farms where data is available, but also at a larger portfolio including wind farms at locations that are not included in the training set. We provided detailed analysis on the forecast performances based on appropriate metrics tailored for probabilistic forecasts.

Our results showed that all the proposed approaches produce calibrated short-term forecasts for individual wind farms. However, we found that modeling spatial dependency is required to achieve calibrated aggregated probabilistic forecasts. Indeed, our case study showed that spatial dependency is important for aggregated properties, and individual forecasts do not reveal this. Moreover, when we simulated from the spatio-temporal model containing an autoregressive term (Model ST+T), we obtained results that are in accordance with our case study, where the proposed models performed equally well for individual forecasts, while aggregated probabilistic forecasts benefit from having a spatio-temporal 
model with the autoregressive term. Model ST+T was introduced due to unsatisfactory reliability for the aggregated forecasts. Hence, evaluating aggregated forecasts can be a tool for investigating and improving models, even when spatially out-of-sample forecasts are the purpose of the modelling. Indeed, results from spatially out-of-sample forecast performances showed that when predicting wind power at new locations that are not included in the training set, having the autoregressive term in the spatio-temporal model improved the forecast performance.

This work was motivated by the need to produce accurate short term probabilistic forecasts at multiple wind farms and lead times, which will ultimately be applied on a national scale. A possible extension of the models described in this work is to include weather forecast information in the linear predictor. This approach usually requires ensemble forecasts to be generated from sophisticated numerical weather prediction (NWP) models and has shown to produce reliable wind power forecasts up to 10 days ahead Taylor et al. [2009].

\section{SUPPLEMENTARY MATERIAL}

The file supplementary.pdf contains the supplementary material for this manuscript. In Section 1 we present tables with summaries of RMSE and CRPS as an extension to the graphical results in Sections 5.1 and 5.2 of this manuscript. Section 2 includes the results of a simulation study based on our case study.

\section{ACKNOWLEDGEMENTS}

The authors are grateful to Energinet.dk (system operator in Denmark) for providing the data and to Robin Girard at MinesParistech, France for checking the quality of the data. The authors also thank the Danish Strategic Council for Strategic Research through the project 5s-Future Electricity Markets (No. 12-132636/DSF), Research Concil of Norway, project 
250362 and CAPES for support. We thank the Associated Editor and the two reviewers who provided valuable comments.

\section{REFERENCES}

Thomas Ackermann. Wind power in power systems. John Wiley \& Sons, 2005.

Marta Blangiardo and Michela Cameletti. Spatial and spatio-temporal Bayesian models with R-INLA. John Wiley \& Sons, 2015.

Leo Breiman and Philip Spector. Submodel selection and evaluation in regression. the x-random case. International Statistical Review/Revue Internationale de Statistique, pages 291-319, 1992.

John Bjørnar Bremnes. Probabilistic wind power forecasts using local quantile regression. Wind Energy, 7 (1):47-54, 2004.

Jochen Bröcker and Leonard A Smith. Scoring probabilistic forecasts: The importance of being proper. Weather and Forecasting, 22(2):382-388, 2007.

Michela Cameletti, Finn Lindgren, Daniel Simpson, and Håvard Rue. Spatio-temporal modeling of particulate matter concentration through the spde approach. AStA Advances in Statistical Analysis, 97(2):109-131, 2013.

João Paulo da Silva Catalão, Hugo Miguel Inácio Pousinho, and Víctor Manuel Fernandes Mendes. Shortterm wind power forecasting in portugal by neural networks and wavelet transform. Renewable Energy, 36(4):1245-1251, 2011.

Daniela Cocchi, Fedele Greco, and Carlo Trivisano. Hierarchical space-time modelling of pm 10 pollution. Atmospheric Environment, 41(3):532-542, 2007.

Noel Cressie. Statistics for spatial data. Terra Nova, 4(5):613-617, 1992.

Noel Cressie. Statistics for spatial data. John Wiley \& Sons, 2015.

Jethro Dowell and Pierre Pinson. Very-short-term probabilistic wind power forecasts by sparse vector autoregression. IEEE Transactions on Smart Grid, 7(2):763-770, 2016.

Alessandro Fassò and Francesco Finazzi. Maximum likelihood estimation of the dynamic coregionalization model with heterotopic data. Environmetrics, 22(6):735-748, 2011.

Ulrich Focken, Matthias Lange, Kai Mönnich, Hans-Peter Waldl, Hans Georg Beyer, and Armin Luig. Shortterm prediction of the aggregated power output of wind farmsa statistical analysis of the reduction of the 
prediction error by spatial smoothing effects. Journal of Wind Engineering and Industrial Aerodynamics, 90(3):231-246, 2002.

Leonard L Garver. Effective load carrying capability of generating units. IEEE Transactions on Power apparatus and Systems, (8):910-919, 1966.

Robin Girard, K Laquaine, and Georges Kariniotakis. Assessment of wind power predictability as a decision factor in the investment phase of wind farms. Applied Energy, 101:609-617, 2013.

Tilmann Gneiting. Quantiles as optimal point forecasts. International Journal of Forecasting, 27(2):197-207, 2011.

Tilmann Gneiting and Peter Guttorp. Continuous parameter spatio-temporal processes. Handbook of Spatial Statistics, 97:427-436, 2010.

Tilmann Gneiting and Adrian E Raftery. Strictly proper scoring rules, prediction, and estimation. Journal of the American Statistical Association, 102(477):359-378, 2007.

Tilmann Gneiting, Kristin Larson, Kenneth Westrick, Marc G Genton, and Eric Aldrich. Calibrated probabilistic forecasting at the stateline wind energy center: The regime-switching space-time method. Journal of the American Statistical Association, 101(475):968-979, 2006.

Tilmann Gneiting, Fadoua Balabdaoui, and Adrian E Raftery. Probabilistic forecasts, calibration and sharpness. Journal of the Royal Statistical Society: Series B (Statistical Methodology), 69(2):243-268, 2007.

Rikke Ingebrigtsen, Finn Lindgren, and Ingelin Steinsland. Spatial models with explanatory variables in the dependence structure. Spatial Statistics, 8:20-38, 2014.

Ron Kohavi et al. A study of cross-validation and bootstrap for accuracy estimation and model selection. In Ijcai, volume 14, pages 1137-1145. Stanford, CA, 1995.

Ada Lau and Patrick McSharry. Approaches for multi-step density forecasts with application to aggregated wind power. The Annals of Applied Statistics, pages 1311-1341, 2010.

Emmanuel Lesaffre, Dimitris Rizopoulos, and Roula Tsonaka. The logistic transform for bounded outcome scores. Biostatistics, 8(1):72-85, 2007.

Finn Lindgren, Håvard Rue, and Johan Lindström. An explicit link between Gaussian fields and Gaussian markov random fields: the stochastic partial differential equation approach. Journal of the Royal Statistical Society: Series B (Statistical Methodology), 73(4):423-498, 2011.

Johan Lindström, Adam Szpiro, Paul D Sampson, Silas Bergen, and Lianne Sheppard. Spatiotemporal: 
An $\mathrm{r}$ package for spatio-temporal modelling of air-pollution. $J$ stat softw (http://cran. rproject. org/web/packages/SpatioTemporal/index. html), 2013.

Johan Lindström, Adam A Szpiro, Paul D Sampson, Assaf P Oron, Mark Richards, Tim V Larson, and Lianne Sheppard. A flexible spatio-temporal model for air pollution with spatial and spatio-temporal covariates. Environmental and ecological statistics, 21(3):411-433, 2014.

Petroula Louka, Georges Galanis, Nils Siebert, Georges Kariniotakis, Petros Katsafados, I Pytharoulis, and G Kallos. Improvements in wind speed forecasts for wind power prediction purposes using kalman filtering. Journal of Wind Engineering and Industrial Aerodynamics, 96(12):2348-2362, 2008.

Manuel A Matos and Ricardo J Bessa. Setting the operating reserve using probabilistic wind power forecasts. IEEE Transactions on Power Systems, 26(2):594-603, 2011.

Facundo Munoz, M Grazia Pennino, David Conesa, Antonio López-Quílez, and José M Bellido. Estimation and prediction of the spatial occurrence of fish species using bayesian latent Gaussian models. Stochastic Environmental Research and Risk Assessment, 27(5):1171-1180, 2013.

Christopher J Paciorek. Bayesian smoothing with Gaussian processes using fourier basis functions in the spectralgp package. Journal of Statistical Software, 19(2):nihpa22751, 2007.

Anthony Papavasiliou and Shmuel S Oren. Multiarea stochastic unit commitment for high wind penetration in a transmission constrained network. Operations Research, 61(3):578-592, 2013.

Pierre Pinson. Very-short-term probabilistic forecasting of wind power with generalized logit-normal distributions. Journal of the Royal Statistical Society: Series C (Applied Statistics), 61(4):555-576, 2012.

Pierre Pinson and George Kariniotakis. Conditional prediction intervals of wind power generation. IEEE Transactions on Power Systems, 25(4):1845-1856, 2010.

Pierre Pinson and Julija Tastu. Discrimination ability of the energy score. Technical report, Technical University of Denmark, 2013.

Sheldon Ross. A first course in probability. Pearson, 2015.

Håvard Rue, Sara Martino, and Nicolas Chopin. Approximate bayesian inference for latent Gaussian models by using integrated nested Laplace approximations. Journal of the Royal Statistical Society: Series B (Statistical Methodology), 71(2):319-392, 2009.

Michael Scheuerer and Thomas M Hamill. Variogram-based proper scoring rules for probabilistic forecasts of multivariate quantities. Monthly Weather Review, 143(4):1321-1334, 2015. 
Julija Tastu, Pierre Pinson, Ewelina Kotwa, Henrik Madsen, and Henrik Aa Nielsen. Spatio-temporal analysis and modeling of short-term wind power forecast errors. Wind Energy, 14(1):43-60, 2011.

James W Taylor, Patrick E McSharry, and Roberto Buizza. Wind power density forecasting using ensemble predictions and time series models. IEEE Transactions on Energy Conversion, 24(3):775-782, 2009.

Thordis L Thorarinsdottir, Michael Scheuerer, and Christopher Heinz. Assessing the calibration of highdimensional ensemble forecasts using rank histograms. Journal of Computational and Graphical Statistics, 25(1):105-122, 2016.

Zoltan Toth, Oliver Talagrand, Guillem Candille, and Yuejian Zhu. Forecast verification: A practitioners guide in atmospheric science, 2003.

J Wang, A Botterud, R Bessa, H Keko, L Carvalho, D Issicaba, J Sumaili, and V Miranda. Wind power forecasting uncertainty and unit commitment. Applied Energy, 88(11):4014-4023, 2011.

Peter Whittle. On stationary processes in the plane. Biometrika, pages 434-449, 1954.

Peter Whittle. Stochastic-processes in several dimensions. Bulletin of the International Statistical Institute, 40(2):974-994, 1963.

Christopher K Wikle and Noel Cressie. A dimension-reduced approach to space-time kalman filtering. Biometrika, 86(4):815-829, 1999. 


\section{FIGURES}

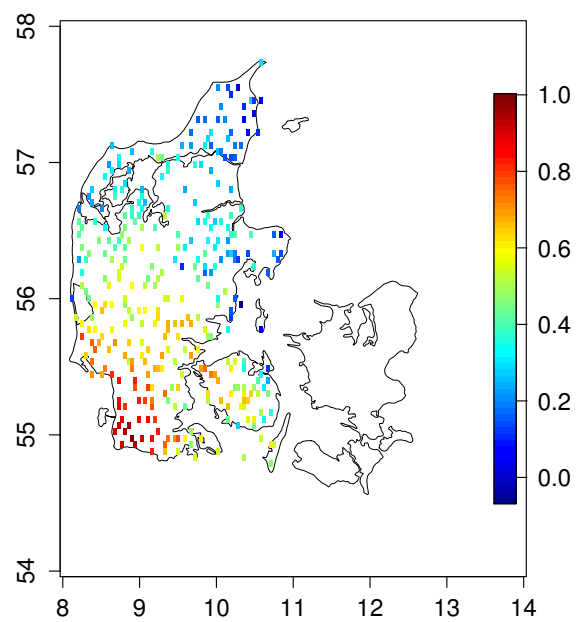

(a)

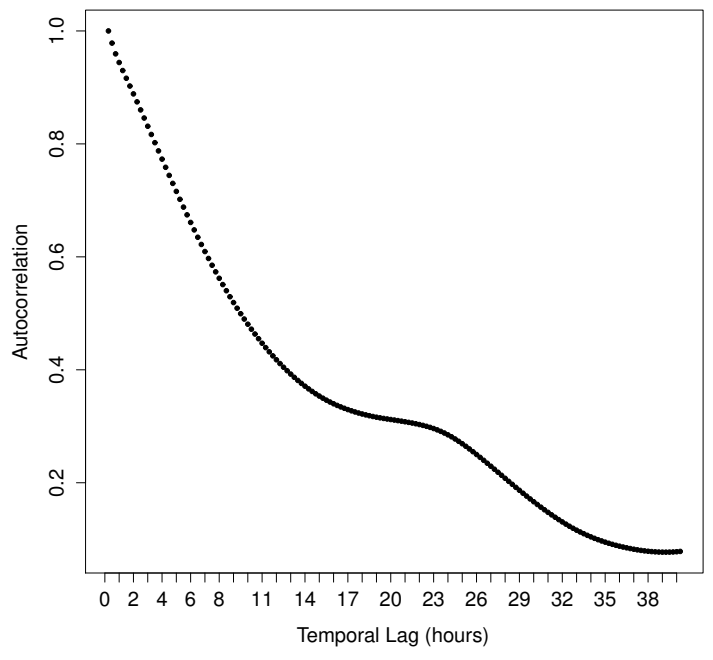

(b)

Figure 1. (a) Map of spatial correlation of wind power production between one wind farm located in the southern part of western Denmark and the remaining wind farms. The correlations between wind farms in a closer proximity are clearly higher than between wind farms that are farther apart. (b) Mean autocorrelation function of wind power production at wind farms located in western Denmark. The autocorrelations decay slowly. 

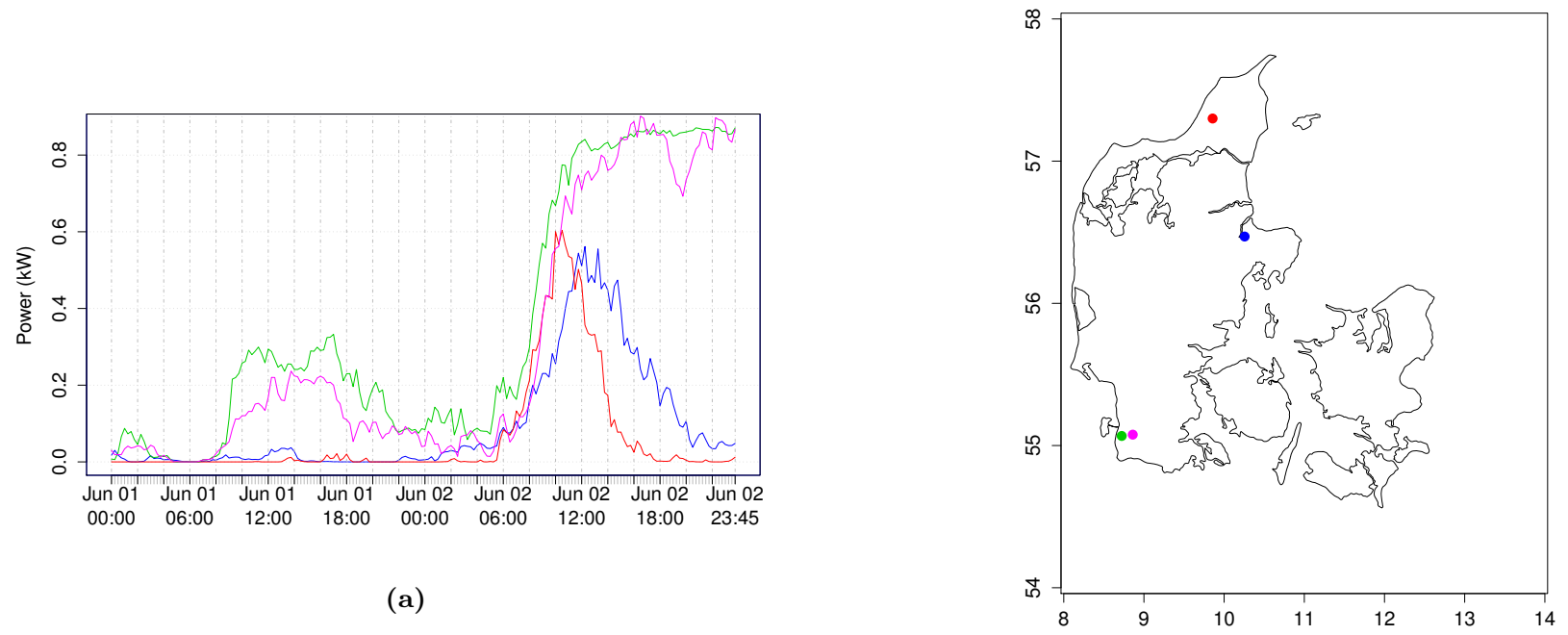

(a)

(b)

Figure 2. (a) 15 min resolution of normalized wind power production at four wind farms from the start of June 1 to the end of June 2. (b) Locations of the four wind farms presented in (a). 


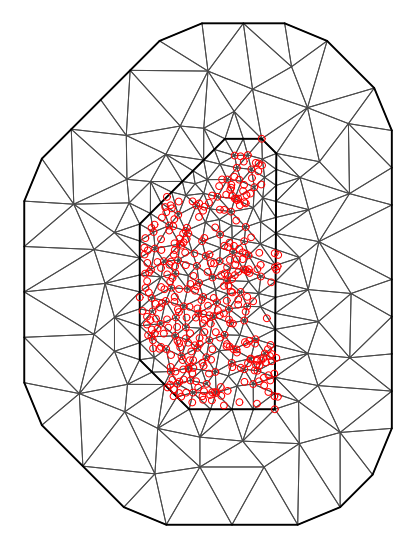

Figure 3. The western Denmark triangulation. The red dots denote the observation locations of the wind power production data. 

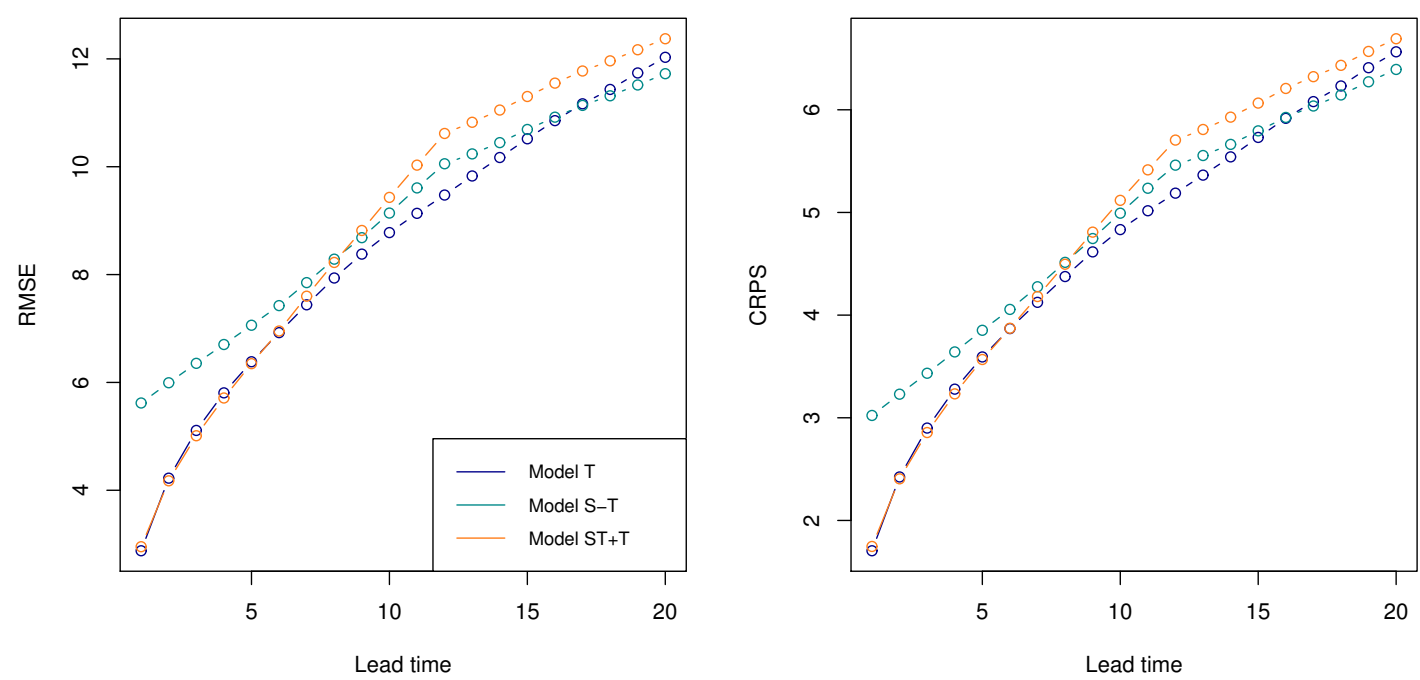

(a)
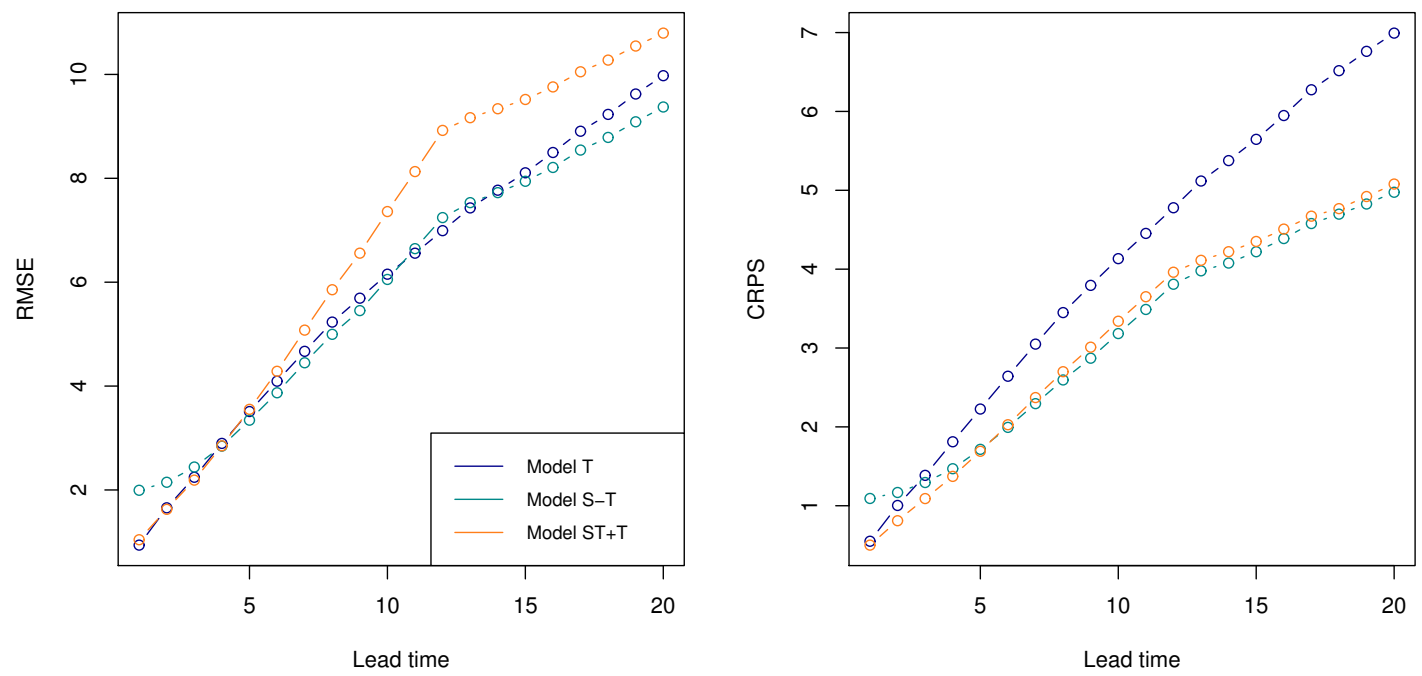

(b)

Figure 4. Mean RMSE and mean CRPS (as \% of nominal power) of spatio-temporal wind power forecasts at lead times $1, \ldots, 20$ (i.e., from 15 minutes up to 5 hours) for Model T (blue), Model S-T (green) and Model ST+T (orange). (a) Forecasts for individual wind farms. (b) Forecasts for aggregated wind farms. 

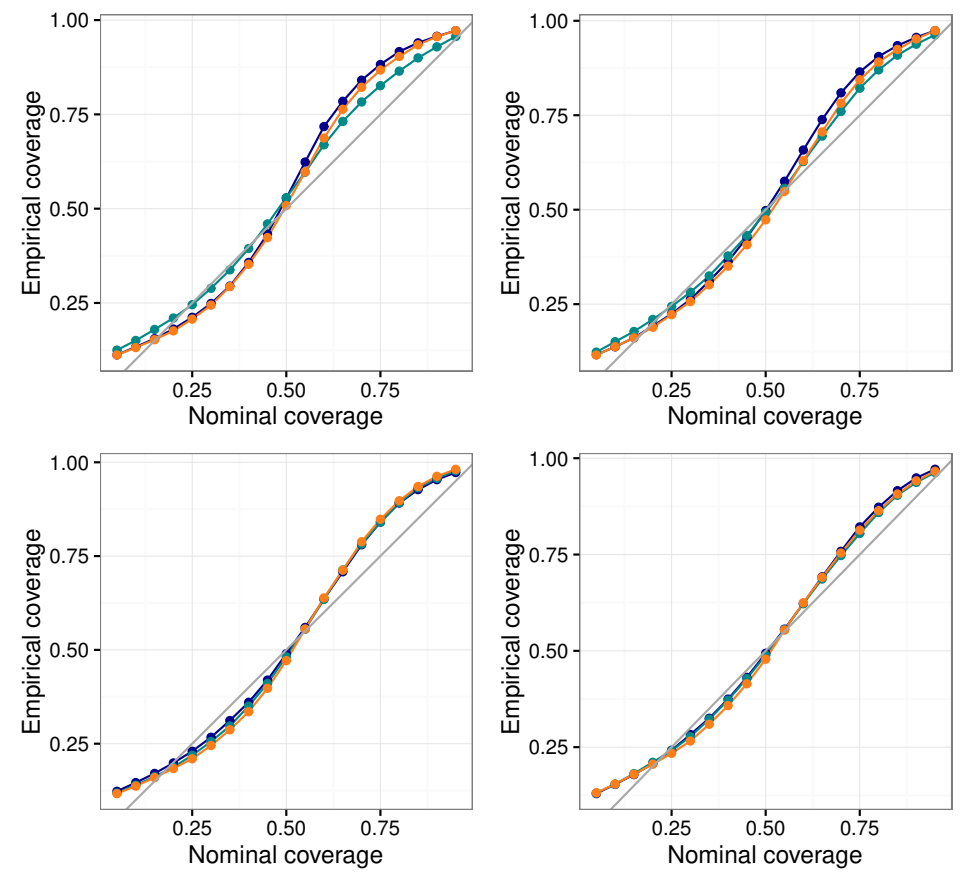

Model

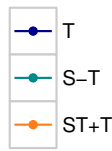

(a)
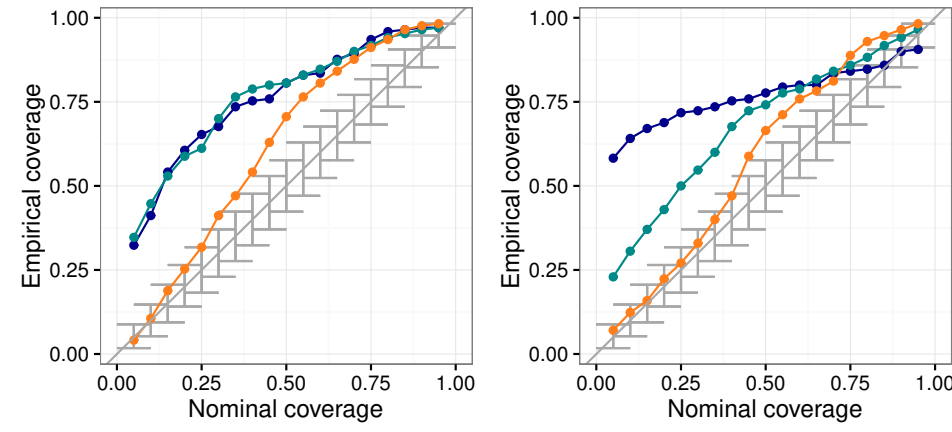

Model
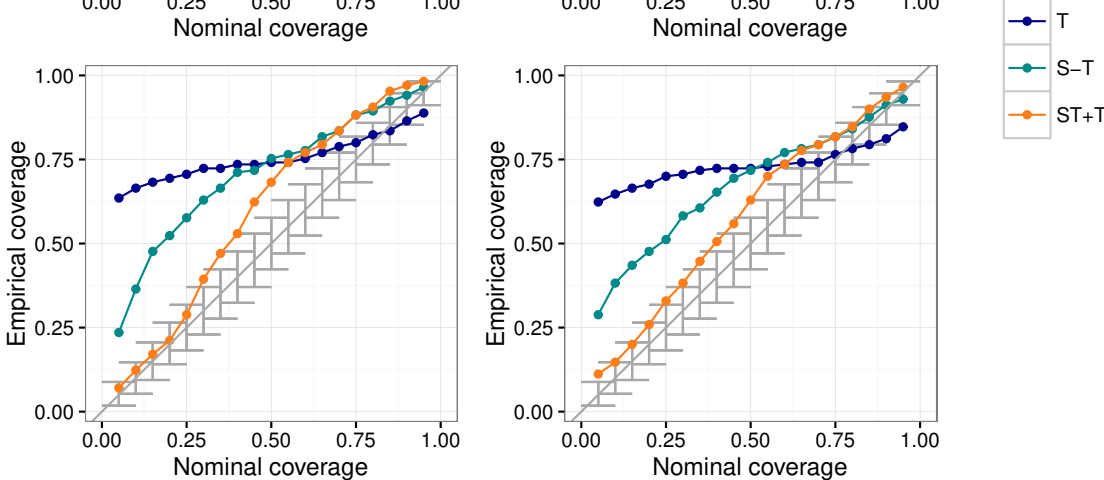

$\rightarrow S T+T$

(b)

Figure 5. Reliability diagram of spatio-temporal wind power forecasts at lead time 1 (Top left), 7 (Top right), 13 (Bottom left) and 19 (Bottom right). The diagrams were calculated using Model T (blue), Model S-T (green) and Model ST+T (orange). (a) Forecasts for individual wind farms. (b) Forecasts for aggregated wind farms. 

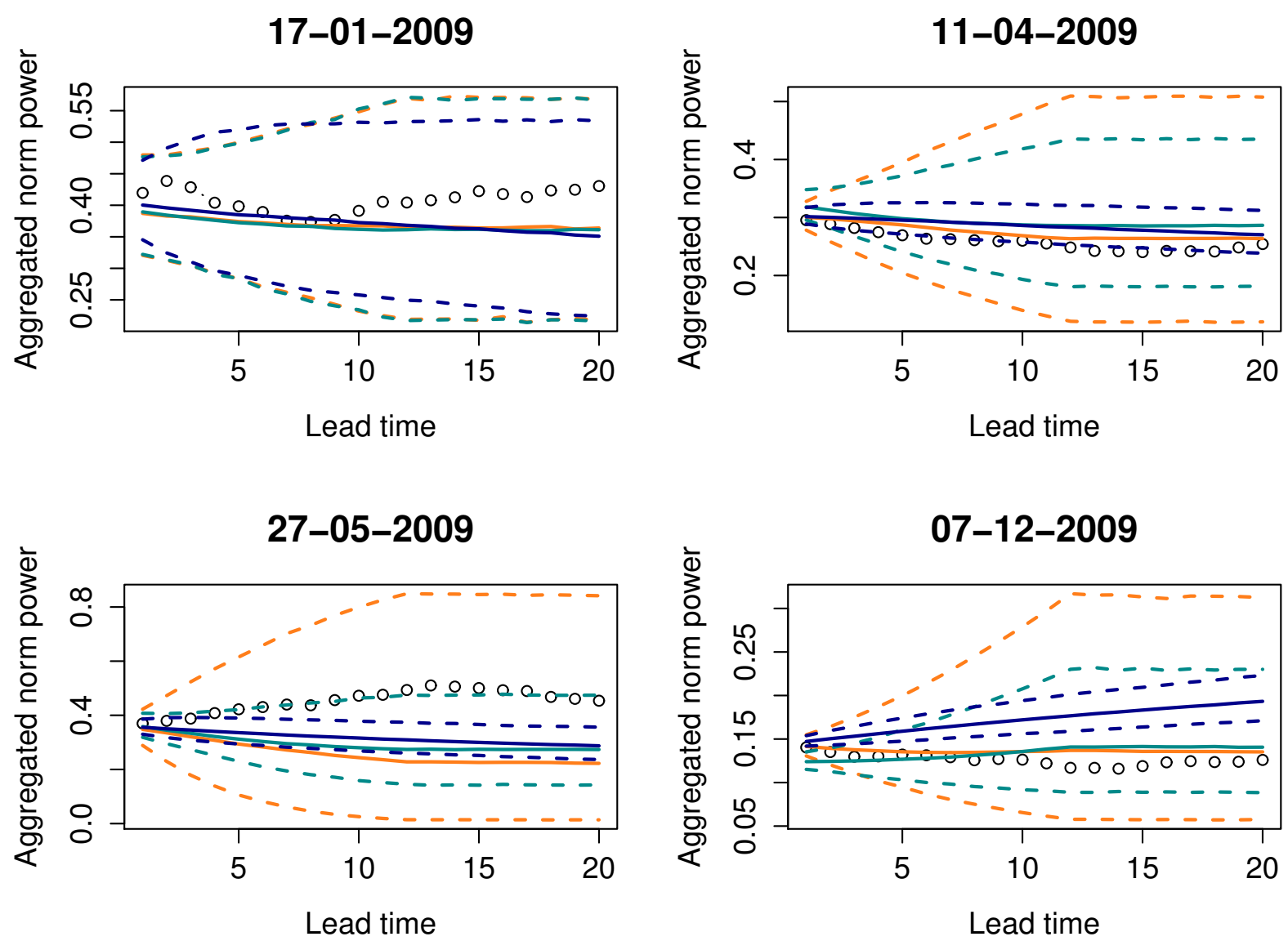

Figure 6. $5 \%$ and $95 \%$ quantiles (dashed lines), as well as the median (solid lines) of the aggregated forecast densities from four different data sets in the training set, together with the actual observed aggregated power produced (circles) at lead times 1-20 (i.e., from 15 minutes up to 5 hours). The forecast densities correspond to Model T (blue), Model S-T (green) and Model ST+T (orange). An example of a data set where all the models have forecast densities that cover the actual aggregated production is shown in the Top left plot. In the Top right plot, the observations lie close to the median of the forecast densities from Model S-T and Model ST+T, but close to the $5 \%$ quantile of the forecast density from Model T. Bottom left and Bottom right plots illustrate cases where Model T has forecast densities that are too narrow and fail to predict the aggregated wind power, while the forecasts from Model ST $+\mathrm{T}$ provide densities that are wide enough to cover the true value at all lead times. 

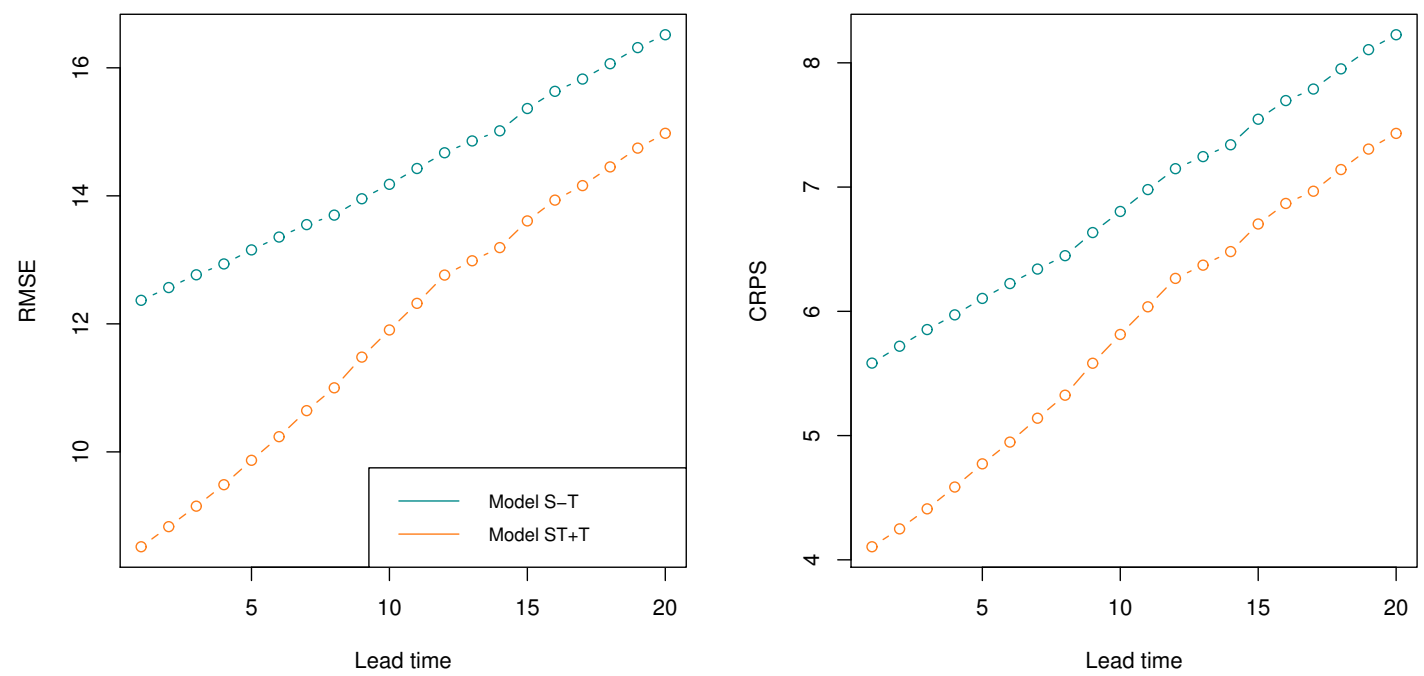

(a)
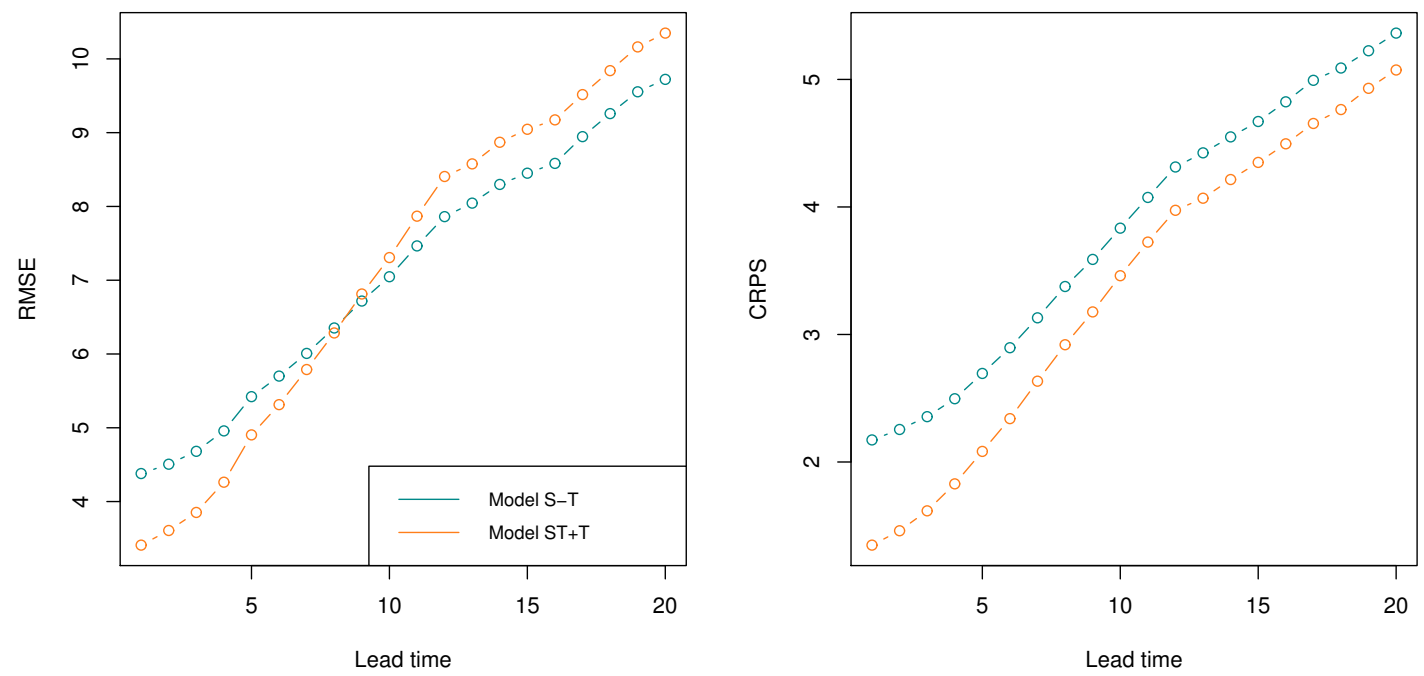

(b)

Figure 7. Mean RMSE and mean CRPS (as \% of nominal power) of spatially out-of-sample wind power forecasts at lead times $1, \ldots, 20$ (i.e., from 15 minutes up to 5 hours) for Model T (blue), Model S-T (green) and Model ST+T (orange). (a) Forecasts for individual wind farms. (b) Forecasts for aggregated wind farms. 

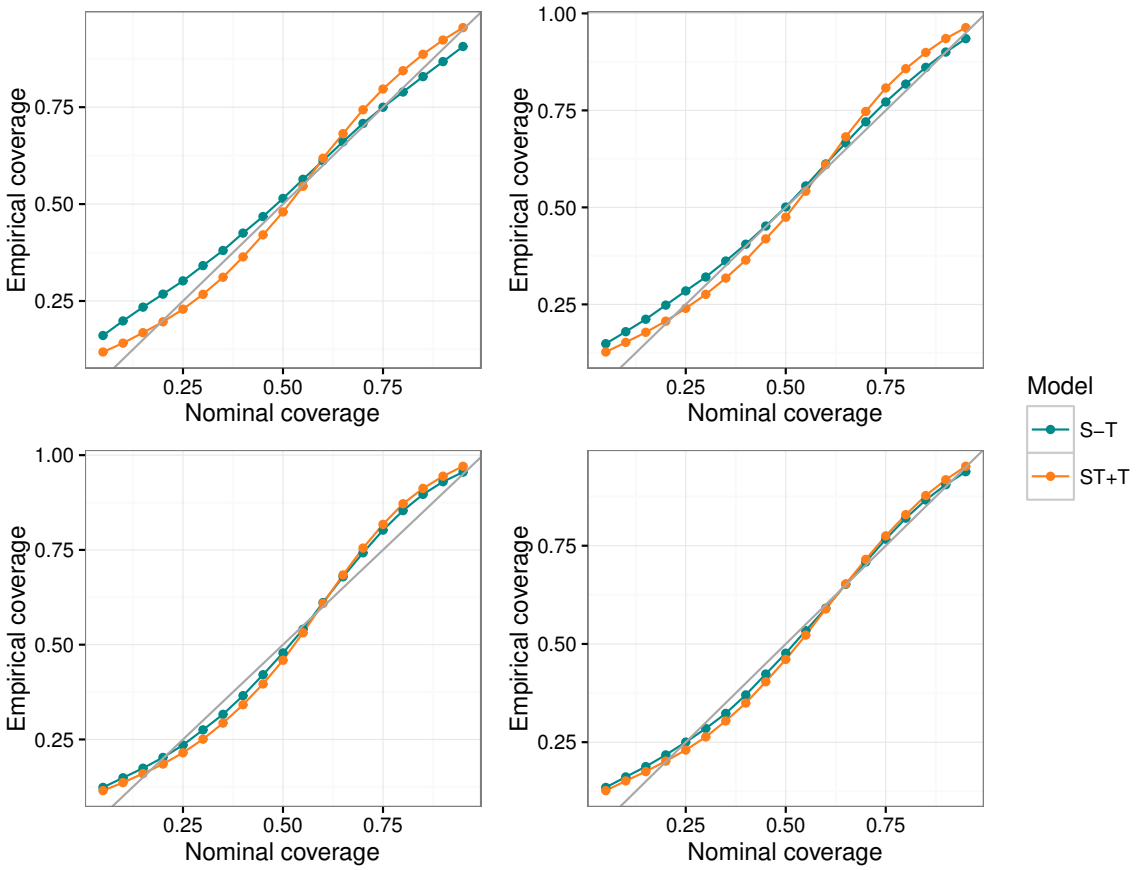

(a)
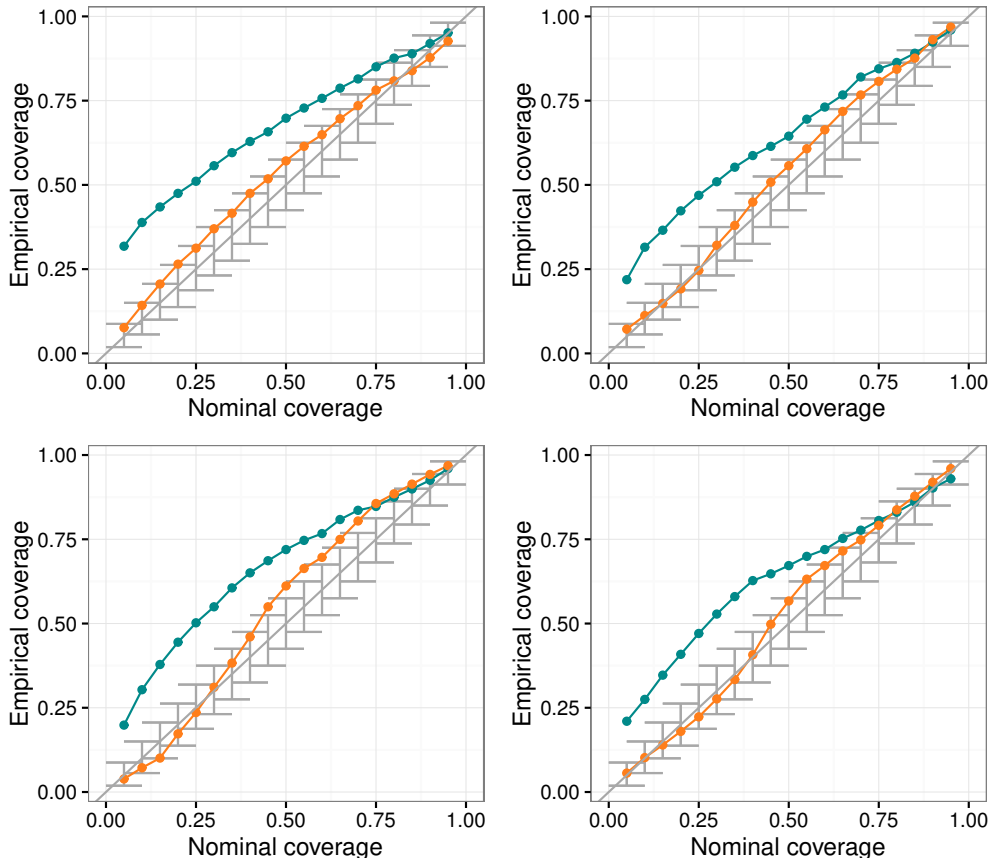

Model
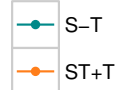

(b)

Figure 8. Reliability diagram of spatially out-of-sample wind power forecasts at lead time 1 (Top left), 7 (Top right), 13 (Bottom left) and 19 (Bottom right). The diagrams were calculated using Model T (blue), Model S-T (green) and Model ST+T (orange). (a) Forecasts for individual wind farms. (b) Forecasts for aggregated wind farms. 